\title{
Expression of L-Type Amino Acid Transporter I is a Predictive Biomarker of Intravesical Recurrence in Patients with Non-Muscle Invasive Bladder
}

\section{Cancer}

\author{
Harutake Sawazaki iD ${ }^{1,2}$ \\ Yuichi Arai ${ }^{3}$ \\ Yuji Ito 4 \\ Kimiya Sato 5 \\ Hitoshi Tsuda ${ }^{5}$ \\ Takashi Yamaga ${ }^{2}$ \\ Hiroyuki Sakurai ${ }^{2}$ \\ 'Department of Urology, Tama-Hokubu \\ Medical Center, Higashimurayama, Japan; \\ ${ }^{2}$ Department of Pharmacology and \\ Toxicology, Kyorin University School of \\ Medicine, Mitaka, Japan; ${ }^{3}$ Department of \\ Urology, National Defense Medical \\ College, Tokorozawa, Japan; \\ ${ }^{4}$ Department of Pathology, Tama-Hokubu \\ Medical Center, Higashimurayama, Japan; \\ ${ }^{5}$ Department of Basic Pathology, National \\ Defense Medical College, Tokorozawa, \\ Japan
}

Correspondence: Harutake Sawazaki Department of Urology, Tama-Hokubu Medical Center, I-7-I Aobacho, Higashimurayama, Tokyo, 189-85I I, Japan Tel +8I-42-396-38II

Fax +8I-42-396-3076

Email harutake_sawazaki@Tokyo-hmt.jp
Purpose: L-type amino acid transporter 1 (LAT1), a $\mathrm{Na}^{+}$-independent amino acid transporter, is highly expressed in various cancer types. We evaluated the prognostic value of LAT1 expression in non-muscle-invasive bladder cancer (NMIBC).

Patients and Methods: We retrospectively reviewed 119 consecutive patients who underwent initial transurethral resection of bladder tumor. Of these, 75 patients with NMIBC were included in this study. Patients were classified into two groups according to the proportion of LAT1-positive cells, as determined by immunohistochemistry. Associations between LAT1 expression and clinicopathological factors were analyzed. Cox multivariate analyses were performed to identify independent predictors of intravesical recurrence (IVR). The LAT1 integrated risk model was compared with the European Organization for Research and Treatment of Cancer (EORTC) risk model to evaluate the predictive ability for IVR based on the c-index.

Results: The median follow-up was 37 months. Twenty-eight patients (37.3\%) had IVR. LAT1 expression was not correlated with any other clinicopathological factors. Patients with high LAT1 expression had a worse IVR-free survival than that of patients with low LAT1 expression $(\mathrm{P}=0.038)$. Cox multivariate analyses indicated that tumor multiplicity and high LAT1 expression were independent predictors of IVR. The LAT1 integrated risk model had a significantly improved performance over the EORTC model for assessing recurrence risk (c-index: 0.695 , improvement: $0.091, \mathrm{P}=0.001$ ). When patients were stratified into three groups according to the score calculated by the LAT1 integrated risk model, the 2-year IVRfree survival rates were $93.3 \%$ in patients with 0 points, $66.9 \%$ for those with 2 points, and $37.5 \%$ for those with 4 points.

Conclusion: High LAT1 expression was an independent predictor of IVR in patients with NMIBC. The LAT1 integrated risk model had good predictability for IVR.

Keywords: non-muscle-invasive bladder cancer, L-type amino acid transporter 1, transurethral resection of bladder tumor, intravesical recurrence

\section{Introduction}

Bladder cancer is the ninth most frequently diagnosed cancer worldwide and ranks 13 th in terms of cancer deaths. ${ }^{1}$ Approximately $75 \%$ of the detected tumors are classified as non-muscle-invasive bladder cancer (NMIBC) at initial diagnosis. ${ }^{2}$ For NMIBC, the mainstay of treatment is transurethral resection of bladder tumor (TURBT) with intravesical bacillus Calmette-Guérin (BCG) instillation or prophylactic intravesical instillation of chemotherapy. ${ }^{3}$ The 5-year recurrence and 
progression rates after treatment for NMIBC are in the ranges of $50 \%$ to $70 \%$ and $10 \%$ to $30 \%$, respectively. ${ }^{3}$ To predict the risk of intravesical recurrence (IVR) in NMIBC patients, the European Organization for Research and Treatment of Cancer (EORTC) GenitoUrinary Cancer Group (GUCG) has developed a scoring system and risk tables. The scoring system is based on the six most relevant clinical and pathological factors (number of tumors, tumor diameter, prior recurrence rate, $\mathrm{Ta} / \mathrm{T} 1$ category, concurrent CIS, and grade) and patients are stratified into four groups based on total scores $(0,1-4$, 5-9, and 10-17) reflecting the probabilities of IVR and progression. Reported probabilities of IVR at 1 year for scores $0,1-4,5-9$, and $10-17$ are $15 \%, 24 \%, 38 \%$, and $61 \%$, respectively. ${ }^{2}$ Although the EORTC risk scoring system is a well-established and valuable tool to predict IVR, the accuracy of this scoring system could be improved. ${ }^{4,5}$ Xylinas et al retrospectively evaluated 4689 patients with NMIBC and found that the EORTC risk scoring model exhibits poor discrimination performance for IVR (c-index $=0.597$ ). ${ }^{6}$ This scoring system relies entirely on tumor characteristics and ignores the tumor biology in individual patients. ${ }^{5}$ To predict clinical outcomes more accurately, the incorporation of effective molecular markers reflecting tumor biology into the scoring system is a potential strategy. ${ }^{5}$

Cancer cells require a large amount of nutrients and amino acids for growth, proliferation, and survival. Uptake is facilitated by the upregulation of amino acid transporters. ${ }^{7}$ L-type amino acid transporter 1 (LAT1) is a $\mathrm{Na}^{+}$-independent amino acid transporter and forms a heterodimer complex with a heavy chain of 4F2 antigen $(4 \mathrm{~F} 2 \mathrm{hc}, \mathrm{CD} 98)$ to perform a transporter function in the plasma membrane. LAT1 transports large neutral amino acids, such as leucine, isoleucine, valine, phenylalanine, tyrosine, tryptophan, methionine, and histidine. Leucine is a neutral amino acid with a bulky side chain and serves as a substrate for protein synthesis. ${ }^{7}$ Leucine also stimulates the mTOR complex 1 (mTORC1) pathway. ${ }^{8}$ LAT1 is highly expressed in proliferating tissues and various cancer types. High LAT1 expression is an independent marker for a poor prognosis in various cancer types, including bile duct adenocarcinoma, hepatocellular carcinoma, ovarian carcinoma, multiple myeloma, and cutaneous melanoma. ${ }^{9-13}$ Maimaiti et al reported that high LAT1 expression is an independent predictor for overall survival in patients with bladder cancer undergoing radical cystectomy. ${ }^{14}$ Nakanishi et al reported that active form of LAT1 (co-expression of LAT1 protein with $4 \mathrm{~F} 2 \mathrm{hc}$ protein) is correlated with overall and disease-free survival based on a univariate analysis of patients with upper tract urothelial carcinoma. ${ }^{15}$ However, the association between LAT1 and IVR in patients with NMIBC has not been evaluated.

In this study, we evaluated the predictive value of LAT1 for the risk of IVR in patients with NMIBC and constructed an IVR risk model based on predictive factors identified by Cox univariate analyses.

\section{Patients and Methods}

We retrospectively reviewed 119 consecutive patients who had undergone TURBT for initial bladder tumor at our institute from April 2014 to April 2018. TURBT was performed according to the standard procedure. To ensure the resection quality, the presence of the detrusor muscle in the specimen was required. A second TUR in patients with T1 tumors was not performed. Cauterization was avoided as much as possible during TURBT to avoid tissue deterioration. Thirty-one patients with muscle invasive bladder cancer, two patients with squamous cell carcinoma, one patient with sarcomatoid variant, one patient with urothelial papilloma, one patient with urothelial dysplasia, and eight patients whose follow-up after TURBT was not performed at our institution were excluded from the analysis. Finally, 75 patients (61 men and 14 women, median age 75 years, range 50 to 94 years) with urothelial carcinoma were included (pTis, $\mathrm{pTa}$, and pT1) (Table 1). Postoperative intravesical instillation of BCG was administered to 23 patients (30.6\%). Pathological characteristics were assessed by a genitourinary pathologist using the 2009 TNM classification. Histological grade was assigned according to the 3-tiered World Health Organization grading system (grade 1-3), namely low grade (grade 1,2) and high grade (grade 3 ). ${ }^{16}$ IVR was monitored by examining each patient every 3 months for the first 2 years postoperatively and every 6 months thereafter. Follow-up investigations consisted of urinary cytology and cystoscopy. This study was approved by the institutional review board of the Tama-Hokubu Medical Center (No. 30-17). 
Table I Patient Characteristics

\begin{tabular}{|c|c|}
\hline Parameter & No. of Cases (\%) \\
\hline Age, mean (median) & 74.7 (75) \\
\hline$\geq 70$ years & $53(70.6)$ \\
\hline$<70$ years & $22(29.3)$ \\
\hline \multicolumn{2}{|l|}{ Gender } \\
\hline Male & 61 (8I.3) \\
\hline Female & $14(18.6)$ \\
\hline \multicolumn{2}{|l|}{ Smoking history } \\
\hline Yes & $56(74.6)$ \\
\hline No & $19(25.3)$ \\
\hline \multicolumn{2}{|l|}{ ECOG performance status } \\
\hline 0 & $60(80.0)$ \\
\hline$\geq 1$ & $15(20.0)$ \\
\hline \multicolumn{2}{|l|}{ Multiplicity } \\
\hline Solitary & $40(53.3)$ \\
\hline Multiple & $35(46.6)$ \\
\hline \multicolumn{2}{|l|}{ Tumor size } \\
\hline$<3 \mathrm{~cm}$ & $62(82.6)$ \\
\hline$\geq 3 \mathrm{~cm}$ & $13(17.3)$ \\
\hline \multicolumn{2}{|l|}{ PT stage } \\
\hline Tis & $3(4.0)$ \\
\hline $\mathrm{Ta}$ & $29(38.6)$ \\
\hline TI & $43(57.3)$ \\
\hline \multicolumn{2}{|l|}{ Grade } \\
\hline Low & $50(66.6)$ \\
\hline High & $25(33.3)$ \\
\hline \multicolumn{2}{|l|}{ Concomitant $\mathrm{CIS}$} \\
\hline Present & $28(37.3)$ \\
\hline Absent & $47(62.6)$ \\
\hline \multicolumn{2}{|l|}{ Previous UTUC } \\
\hline Yes & $3(4.0)$ \\
\hline No & $72(96.0)$ \\
\hline
\end{tabular}

Abbreviations: ECOG, Eastern Co-operative Oncology Group; CIS, carcinoma in situ; UTUC, upper tract urothelial carcinoma.

\section{Immunohistochemistry}

Immunohistochemistry was performed using $4-\mu \mathrm{m}$-thick sections cut from formalin-fixed, paraffin-embedded pathology blocks. Immunohistochemistry was performed as previously described. ${ }^{11}$ The primary antibody was a rabbit polyclonal antibody for LAT1 (dilution 1:800, NB100-734; Novus Biologicals, Littleton, CO). Human testicular tissue was used as positive control. Sections were incubated without the primary antibody (with only phosphate-buffered saline) as a negative control. Two investigators (H.S. and K.S.) blinded to the patients' clinical course independently evaluated the stained slides. The levels of LAT1 expression were assessed according to the ratio of carcinoma cells with positive staining to all carcinoma cells on the slide, regardless of the immunoreaction intensity. Expression levels were classified into three groups: score 1 if $\leq 10 \%$ of the tumor area was stained; score 2 if $11-50 \%$ of the tumor area was stained; and score 3 if $\geq 51 \%$ of the tumor area was stained (Figure 1). The LAT1 expression status was finally categorized into two groups: low expression (score 1) and high expression (score 2 or 3), different from a previous study in which LAT1 expression was classified into four groups. ${ }^{11}$

\section{Statistical Analysis}

The $\chi^{2}$ test or Fisher's extract test was used to evaluate the correlations between LAT1 expression and clinicopathological factors. Survival curves were drawn with the Kaplan-Meier method, and differences were analyzed by the Log rank test. Univariate and multivariate Cox proportional hazard regression models were used to identify significant prognostic indicators. To estimate the IVR risk more effectively, we constructed an IVR risk model consisting of statistically significant parameters identified by Cox univariate analyses.

The constructed model was assessed by using receiver operation characteristic (ROC) curves and was compared with the EORTC risk model by using Harrell's concordance index (c-index). P $<0.05$ was considered statistically significant. All tests were implemented in SPSS ${ }^{\circledR}$ version 26 (IBM Corp., Armonk, NY).

\section{Results}

The median follow-up was 37 months (range 9-70 months). Twenty-eight patients (37.3\%) had IVR. High LAT1 expression was detected in 41 tumors (54.6\%). LAT1 expression was not correlated with any other clinicopathological factors (Table 2).

Kaplan-Meier survival curves were drawn for the patient groups with NMIBC with score 1, score 2, and score 3 (Figure 2A). IVR-free survival rates tended to decrease as the LAT1 score increased. Patients with high LAT1 expression had significantly worse IVR-free survival than that of patients with low LAT1 expression $(P=$ 

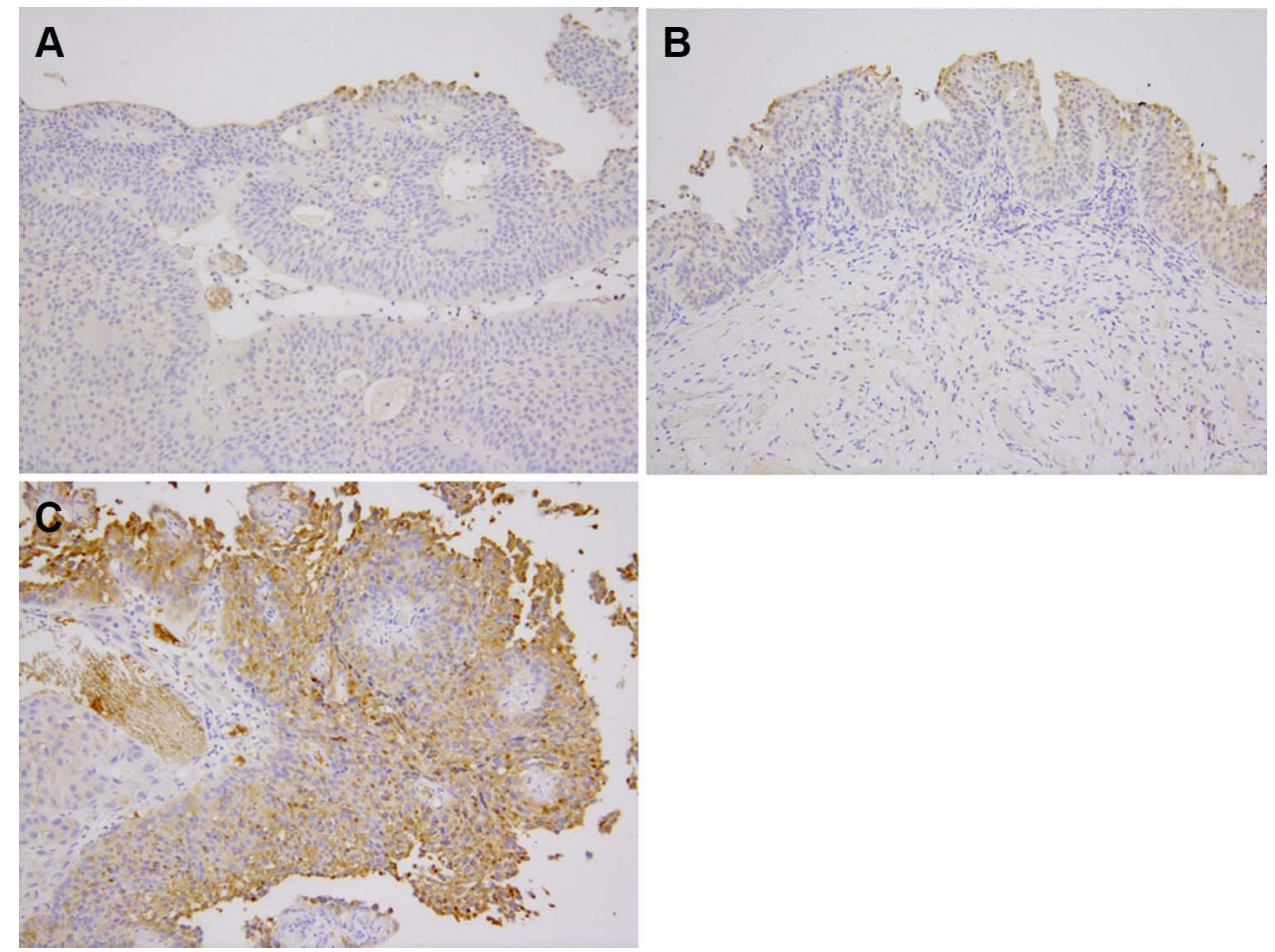

Figure I Immunohistochemical staining of LATI in bladder urothelial carcinoma. Based on the staining degree, LATI expression was classified into three levels. (A) Score I, where $\leq 10 \%$ of the tumor area is stained. (B) Score 2, where II-50\% of the tumor area is stained. (C) Score 3 , where $\geq 51 \%$ of the tumor area is stained. Original magnification, $\times 200$.

0.038) (Figure 2B). A univariate analysis revealed that tumor multiplicity and high LAT1 expression were significant factors for IVR. A Cox multivariate analysis showed that tumor multiplicity and high LAT1 expression are independent risk factors for IVR (Table 3).

To establish the LAT1 integrated risk model, LAT1 expression and tumor multiplicity were used. Low LAT1 expression and high LAT1 expression were assigned 0 and 2 points, respectively. Tumor multiplicity no and yes were assigned 0 and 2 points, respectively (Table 4). Finally, risk points for LAT1 expression and tumor multiplicity were summed to obtain total points. In this cohort, total points were 0 in 15 patients, 2 in 44 , and 4 in 16 . ROC AUC values were obtained to assess the clinical performance of the LAT1 integrated risk model and EORTC risk model. The LAT1 integrated risk model had an AUC of 0.695, compared to 0.604 for the EORTC risk model (Figure 3). Compared to the EORTC risk model, the LAT1 integrated risk model showed a significantly improved discriminative performance $(\mathrm{c}$-index $=0.695$, increase: $0.091,95 \% \mathrm{CI}: 0.575-0.815, P=0.001)$. This cohort was stratified into three categories according to total points calculated by the LAT1 integrated risk model. The 2-year IVR-free survival rates were $93.3 \%$ in patients with 0 points, $66.9 \%$ in patients with 2 points, and $37.5 \%$ in patients with 4 points (Figure 4 ).

\section{Discussion}

This study revealed that LAT1 expression is a risk factor for IVR in patients with NMIBC undergoing TURBT. Patients with high LAT1 expression had a significantly higher IVR rate than that of patients with low LAT1 expression. High LAT1 expression was an independent predictor of IVR. The LAT1 integrated risk model showed an improved predictability for IVR.

LAT1 provides essential amino acids required for protein synthesis in cancer cells and facilitates cancer cell growth by the stimulation of the mTOR signaling pathway. LAT1 is highly expressed in various cancer types and high LAT1 expression has been identified as an independent marker of a poor prognosis. Lu conducted a systematic review and meta-analysis to assess the prognostic value of LAT1 in solid cancers. ${ }^{17}$ A total of 4579 cases were analyzed from 35 qualified studies involving 16 cancer 
Table 2 Correlations Between LATI Expression and Clinicopathological Parameters in 75 Patients with Non-Muscle Invasive Bladder Cancer Undergoing Transurethral Resection of Bladder Tumor

\begin{tabular}{|c|c|c|c|c|}
\hline & No. of Cases (\%) & Low LATI $(n=34)$ & High LATI $(n=4 I)$ & $P$-value \\
\hline & Total & & & \\
\hline Age (years), mean \pm SD & $74.7 \pm 8.7$ & $75.7 \pm 8.1$ & $74.0 \pm 9.1$ & 0.40 \\
\hline Gender & & & & 0.69 \\
\hline Male & $61(81.3)$ & $27(79.4)$ & $34(82.9)$ & \\
\hline Female & $14(18.6)$ & $7(20.5)$ & $7(17.0)$ & \\
\hline Smoking history & & & & 0.83 \\
\hline Yes & $56(74.6)$ & $25(73.5)$ & $31(75.6)$ & \\
\hline No & $19(25.3)$ & $9(26.4)$ & $10(24.3)$ & \\
\hline Multiplicity & & & & 0.14 \\
\hline Solitary & $40(53.3)$ & $15(44.1)$ & $25(60.9)$ & \\
\hline Multiple & $35(46.6)$ & $19(55.8)$ & $16(39.0)$ & \\
\hline Tumor size & & & & 0.057 \\
\hline$<3 \mathrm{~cm}$ & $62(82.6)$ & $25(73.5)$ & $37(90.2)$ & \\
\hline$\geq 3 \mathrm{~cm}$ & $13(17.3)$ & $9(26.4)$ & $4(9.7)$ & \\
\hline PT stage & & & & 0.27 \\
\hline Tis & $3(4.0)$ & $0(0)$ & $3(7.3)$ & \\
\hline $\mathrm{Ta}$ & $29(38.6)$ & $14(4 \mid .1)$ & $15(36.5)$ & \\
\hline TI & $43(57.3)$ & $20(58.8)$ & $23(56.0)$ & \\
\hline Grade & & & & 0.07 \\
\hline Low & $50(66.6)$ & $19(55.8)$ & $31(75.6)$ & \\
\hline High & $25(33.3)$ & $15(44.1)$ & $10(24.3)$ & \\
\hline Concomitant CIS & & & & 0.53 \\
\hline Present & $28(37.3)$ & $14(41.1)$ & $14(34.1)$ & \\
\hline Absent & $47(62.6)$ & $20(58.8)$ & $27(65.8)$ & \\
\hline Intravesical BCG therapy & & & & 0.42 \\
\hline Yes & $23(30.6)$ & $12(35.2)$ & II (26.8) & \\
\hline No & $52(69.3)$ & $22(64.7)$ & $30(73.1)$ & \\
\hline
\end{tabular}

Abbreviations: BCG, bacillus Calmette-Guérin; CIS, carcinoma in situ; SD, standard deviation.

types. In patients with solid cancers, elevated LAT1 expression was associated with a poor overall survival, disease-free survival, and progression-free survival. This meta-analysis revealed the reliability and potential prognostic value of LAT1 protein expression in patients with various solid cancers. ${ }^{17} \mathrm{~A}$ few studies assessed the value of LAT1 expression in urothelial carcinoma. Maimaiti et al reported that LAT1 expression is an independent predictor for overall survival in patients with bladder cancer undergoing radical cystectomy $(\mathrm{HR}=3.14,95 \% \mathrm{CI}: 1.20-11.23$, $P=0.0204)$. High LAT1 expression was significantly associated with the tumor grade, advanced pT stage, LDH level, and neutrophil-lymphocyte ratio. ${ }^{14}$ Nakanishi et al reported that the active form of LAT1 is significantly associated with overall and disease-free survival in upper tract urothelial carcinoma based on a univariate analysis. ${ }^{15}$ Our results indicated that high LAT1 expression is an independent predictor of IVR in patients with NMIBC. Different from the aforementioned two studies of LAT1 in bladder or upper tract urothelial cancers, there were no correlations between LAT1 expression levels and any clinicopathological factors in this study.

The EORTC risk scoring model is based on wellcontrolled, prospective, randomized clinical trials and is the most widely used model. However, previous studies have reported that this risk scoring model has suboptimal discriminatory power to predict IVR in patients with NMIBC. ${ }^{4,6}$ Xylinas et al retrospectively assessed the 

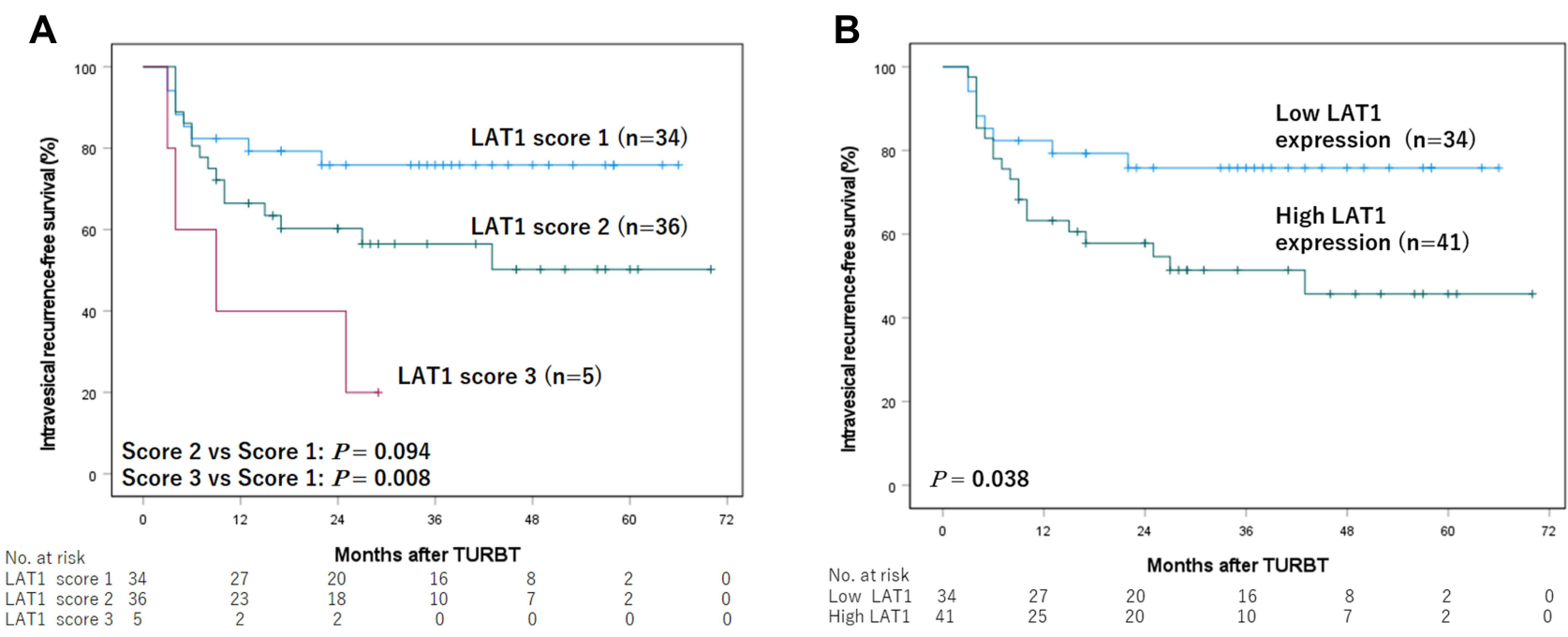

Figure 2 (A) Kaplan-Meier survival curves for groups with NMIBC with various LATI expression scores. (B) Kaplan-Meier curves stratified by LATI expression status (low or high).

discrimination of the EORTC risk model in 4689 patients with NMIBC but found a poor discrimination performance for IVR (c-index $=0.597){ }^{6}$ This risk scoring system does not consider the biological background of individual tumors. ${ }^{5}$ To predict clinical outcomes in individual patients, an effective molecular marker is needed. Several researchers have attempted to use molecular markers (Ki67, FGFR3 mutation, p53, etc.) as prognostic factors to predict outcomes in patients with NMIBC. ${ }^{18}$ Nonetheless, these molecular markers have shown heterogenous results and are not sufficiently validated for use in clinical practice. Our results revealed that high LAT1 expression is an independent predictor of IVR. LAT1 protein expression may be a predictive molecular marker to estimate IVR in individual patients with NMIBC. The LAT1 integrated risk model improved the discriminatory performance for assessing the recurrence risk. Most recurrence occurred within 12 months in patients with 4 points, suggesting that these patients should be followed up more closely than those with 0 or 2 points. The risk stratification model may provide accurate information regarding IVR for patient counseling and follow-up scheduling. LAT1 is a potential therapeutic target for NMIBC with high LAT expression.

Table 3 Univariate and Multivariate Cox Proportional Hazard Regression Analysis of Intravesical Recurrence-Free Survival

\begin{tabular}{|c|c|c|c|c|c|c|}
\hline \multirow[t]{2}{*}{ Variables } & Univariate & \multirow[b]{2}{*}{ HR } & \multirow[b]{2}{*}{$95 \% \mathrm{Cl}$} & Multivariate & \multirow[b]{2}{*}{ HR } & \multirow[b]{2}{*}{$95 \% \mathrm{Cl}$} \\
\hline & $P$-value & & & $P$-value & & \\
\hline Age & 0.49 & 1.01 & $0.97-1.06$ & & & \\
\hline Gender (male vs female) 0.952 & 0.94 & 0.96 & $0.36-2.54$ & & & \\
\hline Smoking history (yes vs no) & 0.32 & 0.61 & $0.23-1.61$ & & & \\
\hline Tumor size $(\geq 3 \mathrm{~cm}$ vs $<3 \mathrm{~cm})$ & 0.14 & 1.90 & $0.80-4.49$ & 0.10 & 3.88 & $0.74-20.2$ \\
\hline Multiplicity (multiple vs single) & $0.02 *$ & 2.37 & I.10-5.08 & $0.02 *$ & 6.62 & $1.32-33.2$ \\
\hline pT stage (pTI vs pTa) & 0.11 & 1.89 & $0.85-4.19$ & 0.10 & 2.53 & $0.14-7.87$ \\
\hline Grade (high vs low) & 0.76 & 0.86 & $0.37-1.95$ & & & \\
\hline CIS (yes vs no) & 0.06 & 0.42 & $0.17-1.03$ & & & \\
\hline EORTC scoring system & 0.056 & 1.13 & $0.99-1.29$ & 0.19 & 0.75 & $0.48-1.16$ \\
\hline LATI expression (high vs low) & $0.04 *$ & 2.29 & $1.01-5.22$ & $0.01 *$ & 2.82 & $1.21-6.58$ \\
\hline
\end{tabular}

Note: *Statistically significant.

Abbreviations: $\mathrm{Cl}$, confidence interval; CIS, carcinoma in situ; EORTC, European Organization for Research and Treatment of Cancer; HR, hazard risk; IVR, intravesical recurrence. 
Table 4 Selected Variables for the LATI Integrated Risk Model

\begin{tabular}{|l|l|l|}
\hline & $\begin{array}{l}\text { No. of Risk Points for } \\
\text { IVR }\end{array}$ & $\begin{array}{l}\text { Odds Ratio (95\% } \\
\mathbf{C I})\end{array}$ \\
\hline $\begin{array}{l}\text { Tumor } \\
\text { multiplicity } \\
\text { Solitary }\end{array}$ & & \\
Multiple & 0 & I (Reference) \\
\hline LATI & $2.37(\mathrm{I} .10-5.08)$ \\
expression & & \\
Low & & I (Reference) \\
High & 2 & 2.29 (I.0I-5.22) \\
\hline
\end{tabular}

Abbreviations: $\mathrm{Cl}$, confidence interval; IVR, intravesical recurrence.

This study had several limitations. First, it was a retrospective single-center study involving a limited number of patients. Although LAT1 expression was a predictive biomarker for IVR in patients with NMIBC, our dataset was small and heterogeneous, making it difficult to obtain definitive conclusions. To verify our findings, a large retrospective series with welldefined inclusion criteria (eg, only BCG-treated highrisk patients) or a multi-institutional prospective trial in a large cohort is required. Second, postoperative intravesical instillation of BCG was administered to 23 patients (30.6\%), and this may have influenced subsequent IVR. Four of these 23 patients developed IVR (17.3\%). One patient (8.3\%) with low LAT1 expression $(\mathrm{n}=12)$ had IVR after BCG therapy. Three patients $(27.2 \%)$ with high LAT1 expression $(\mathrm{n}=11)$ had IVR after BCG therapy. IVR rates after BCG instillation may increase in relation to the LAT1 expression status. Third, the 5-year rates of progression after treatment for NMIBC are in the range of $10 \%$ to $30 \%$; however, this study did not investigate the relationship between LAT1 expression and disease progression. Because only 2 of 75 patients showed disease progression, it was not possible to evaluate the relationship between LAT1 expression and disease progression or overall survival.

\section{Conclusions}

LAT1 expression evaluated immunohistochemically was a predictive biomarker for IVR in patients with NMIBC undergoing TURBT. The newly established LAT1 integrated risk model improved the discriminatory performance for assessing the recurrence risk. A large retrospective series with well-defined inclusion criteria or a multi-institutional prospective trial in a large cohort is required to clarify the impact of LAT1 on IVR in patients with NMIBC.

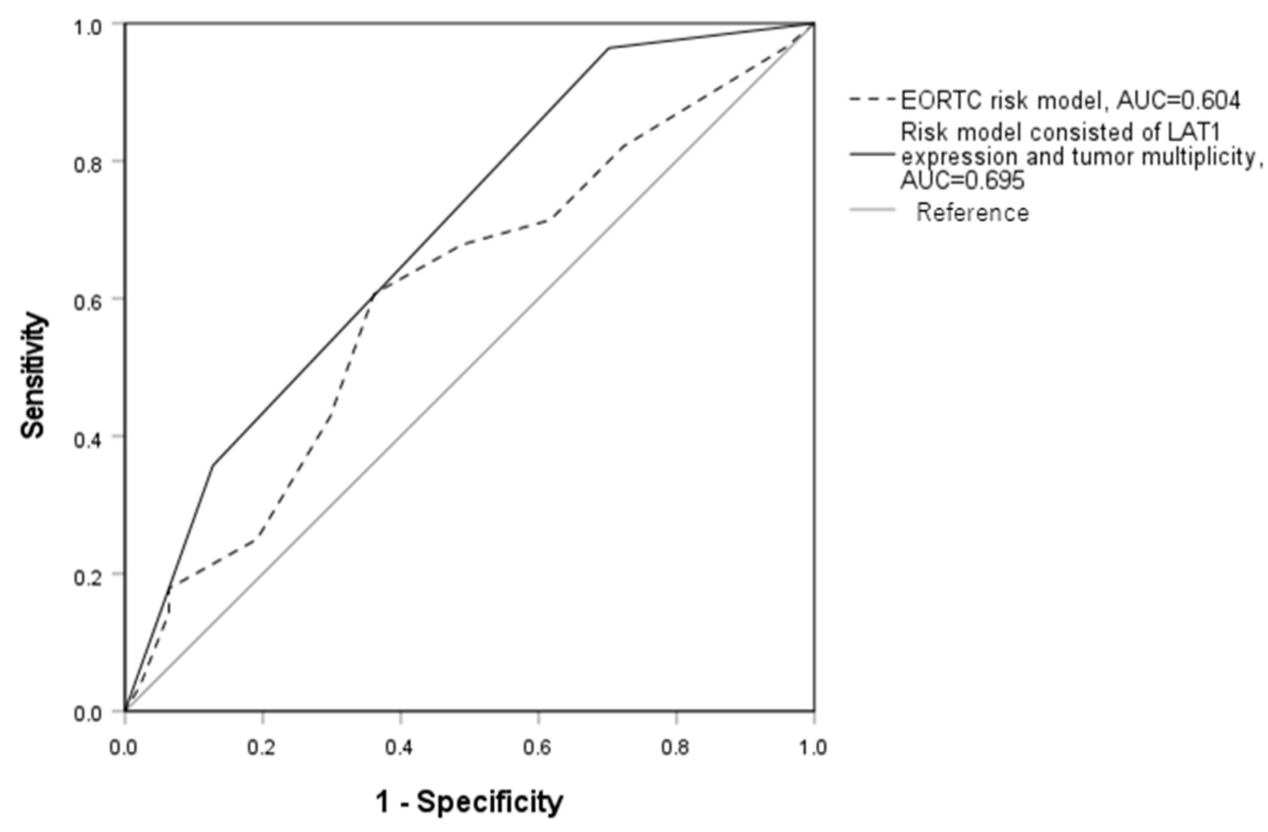

Figure 3 Predictive performance of the EORTC risk scoring model and LATI integrated risk model for intravesical recurrence (IVR) in patients with NMIBC based on AUC values. 


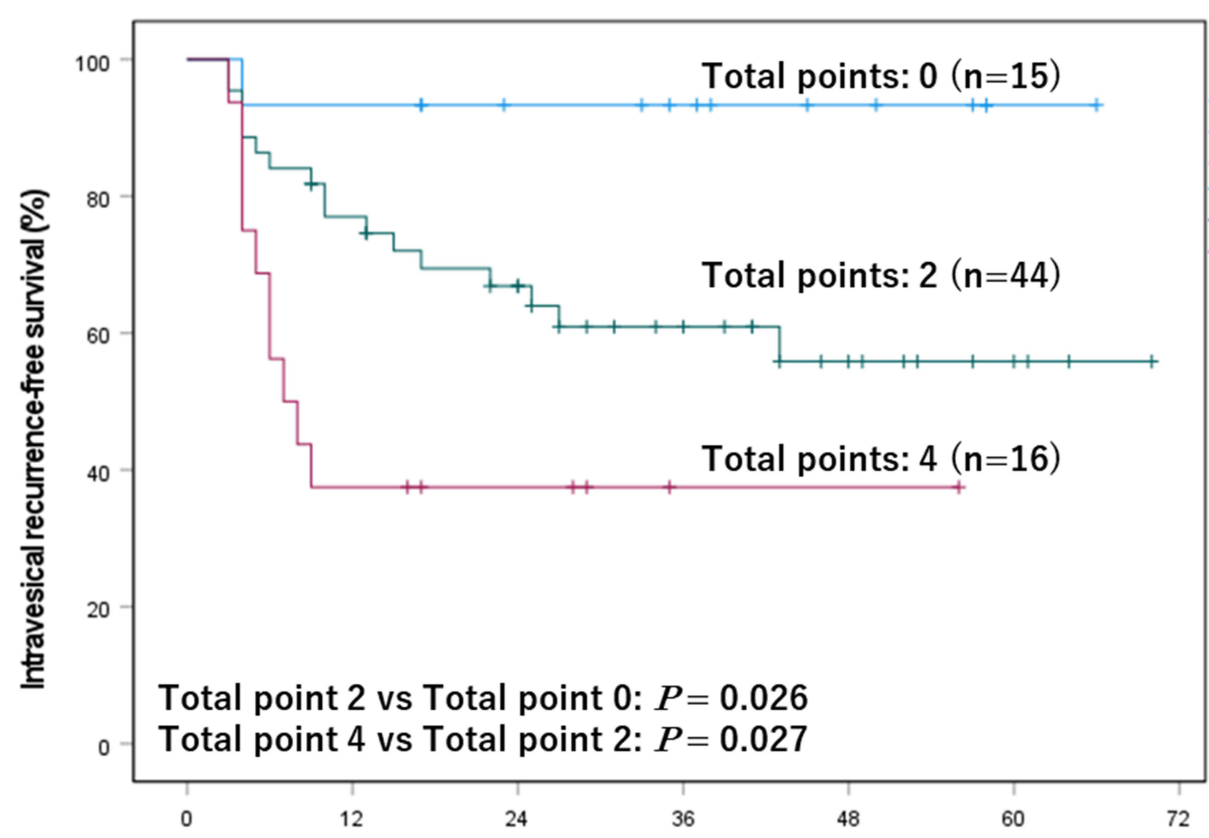

No. at risk

Total point 0

Total point 4

$\begin{array}{cc}15 & 13 \\ 44 & 32 \\ 16 & 6\end{array}$

\begin{tabular}{ccc}
\multicolumn{3}{c}{ Months after TURBT } \\
11 & 9 & 6 \\
24 & 15 & 8 \\
4 & 1 & 0
\end{tabular}

Figure 4 Kaplan-Meier curves for intravesical recurrence (IVR)-free survival of patients with 0, 2, and 4 total points.

\section{Abbreviations}

BCG, bacillus Calmette-Guérin; CIS, carcinoma in situ; EORTC, European Organization for Research and Treatment of Cancer; IVR, intravesical recurrence; LAT1, L-type amino acid transporter 1; NMIBC, non-muscleinvasive bladder cancer; TURBT, transurethral resection of bladder tumor.

\section{Ethical Standards}

All procedures performed in this study involving human participants were in accordance with the ethical standards of the institutional and/or national research committee and with the 1964 Helsinki Declaration and its later amendments or comparable ethical standards. This study was approved by the appropriate ethics committee.

\section{Informed Consent}

No informed consent was obtained from human participants because all data were anonymized.

\section{Acknowledgments}

I would like to thank H. Tsuda and H. Sakurai for useful discussions. I am grateful to K. Sato and Y. Ito for technical assistance with the experiments.

\section{Funding}

There is no funding to report.

\section{Disclosure}

The authors report no conflicts of interest in this work.

\section{References}

1. Antoni S, Ferlay J, Soerjomataram I, et al. Bladder cancer incidence and mortality: a global overview and recent trends. Eur Urol. 2017;71 (1):96-108. doi:10.1016/j.eururo.2016.06.010

2. Babjuk M, Burger M, Comperat EM, et al. European association of urology guidelines on non-muscle invasive bladder cancer (TaT1 and carcinoma in situ) - 2019 update. Eur Urol. 2019;76(5):639-657. doi:10.1016/j.eururo.2019.08.016

3. Kamat AM, Hahn NM, Efstathiou JA, et al. Bladder cancer. Lancet. 2016;388(10061):2796-2810. doi:10.1016/S0140-6736(16)30512-8

4. Fukuokaya W, Kimura T, Miki J, et al. Red cell distribution width predicts time to recurrence in patients with primary non-muscleinvasive bladder cancer and improves the accuracy of the EORTC scoring system. Urol Oncol. 2020;38(7):638.e15-638.e23. doi:10.1016/j.urolonc.2020.01.016

5. Juracek J, Stanik M, Vesela P, et al. Tumor expression of miR-34a-3p is an independent predictor of recurrence in non-muscle-invasive bladder cancer and promising additional factor to improve predictive value of EORTC nomogram. Urol Oncol. 2019;37:184.e1-184.e7. doi:10.1016/j.urolonc.2018.10.014

6. Xylinas E, Kent M, Kluth L, et al. Accuracy of the EORTC risk tables and of the CUERO scoring model to predict outcomes in non-muscleinvasive urothelial carcinoma of the bladder. Br J Cancer. 2013;109 (6):1460-1466. doi:10.1038/bjc.2013.372 
7. Zhao Y, Wang L, Pan J. The role of L-type amino acid transporter 1 in human tumors. Intractable Rare Dis Res. 2015;4(4):165-169. doi:10.5582/irdr.2015.01024

8. Salisbury TB, Arthur S. The regulation and function of the L-type amino acid transporter 1 (LAT1) in cancer. Int J Mol Sci. 2018;19 (8):2373. doi:10.3390/ijms19082373

9. Yanagisawa N, Hana K, Nakada N, et al. High expression of L-type amino acid transporter 1 as a prognostic marker in bile duct adenocarcinoma. Cancer Med. 2014;3(5):1246-1255. doi:10.1002/cam4.272

10. Namikawa M, Kakizaki S, Kaira K, et al. Expression of amino acid transporters (LAT1, ASCT2 and $\mathrm{xCT}$ ) as clinical significance in hepatocellular carcinoma. Hepatol Res. 2015;45(9):1014-1022. doi:10.1111/hepr.12431

11. Sato K, Miyamoto M, Takano M, et al. Significant relationship between the LAT1 expression pattern and chemoresistance in ovarian clear cell carcinoma. Virchows Arch. 2019;474(6):701-710. doi: 10.1007/s00428-019-02520-0

12. Isoda A, Kaira K, Iwashina M, et al. Expression of L-type amino acid transporter 1 (LAT1) as a prognostic and therapeutic indicator in multiple myeloma. Cancer Sci. 2014;105(11):1496-1502. doi:10.1111/cas.12529
13. Shimizu A, Kaira K, Kato M, et al. Prognostic significance of L-type amino acid transporter 1 (LAT1) expression in cutaneous melanoma. Melanoma Res. 2015;25(5):399-405. doi:10.1097/CMR.00000 00000000181

14. Maimaiti M, Sakamoto S, Yamada Y, et al. Expression of L-type amino acid transporter 1 as a molecular target for prognostic and therapeutic indicators in bladder carcinoma. Sci Rep. 2020;10 (1):1292. doi:10.1038/s41598-020-58136-x

15. Nakanishi K, Ogata S, Matsuo H, et al. Expression of LAT1 predicts risk of progression of transitional cell carcinoma of the upper urinary tract. Virchows Arch. 2007;451:681-690. doi:10.1007/s00428-007-0457-9

16. Miyazaki Y, Kosaka T, Mikami S, et al. The prognostic significance of Vasohibin-1 expression in patients with upper tract urothelial carcinoma. Clin Cancer Res. 2012;18:4145-4153. doi:10.1158/ 1078-0432.CCR-12-0073

17. Lu J, Li P, Yang Y, et al. Prognostic value of LAT-1 status in solid cancer: a systematic review and meta-analysis. PLoS One. 2020;15 (5):e0233629. doi:10.1371/journal.pone.0233629

18. Isharwal S, Konety B. Non-muscle invasive bladder cancer risk stratification. Indian J Urol. 2015;31:289-296. doi:10.4103/09701591.166445

\section{Publish your work in this journal}

Research and Reports in Urology is an international, peer-reviewed, open access journal publishing original research, reports, editorials, reviews and commentaries on all aspects of adult and pediatric urology in the clinic and laboratory including the following topics Pathology, pathophysiology of urological disease; Investigation and treatment of urological disease; Pharmacology of drugs used for the treatment of urological disease. The manuscript management system is completely online and includes a very quick and fair peer-review system, which is all easy to use. Visit http://www.dovepress.com/ testimonials.php to read real quotes from published authors. 